\title{
A dietary pattern rich in lignans, quercetin and resveratrol decreases the risk of oesophageal cancer
}

\author{
Yulan Lin ${ }^{1 *}$, Agneta Yngve ${ }^{2}$, Jesper Lagergren ${ }^{1,3}$ and Yunxia Lu ${ }^{1,4}$ \\ ${ }^{1}$ Unit of Upper Gastrointestinal Research, Department of Molecular Medicine and Surgery, Karolinska Institutet, \\ Norra Stationsgatan 67, 2nd floor, 17176 Stockbolm, Sweden \\ ${ }^{2}$ School of Hospitality, Culinary Arts and Meal Sciences, Örebro University, Grythyttan Campus, Örebro, Sweden \\ ${ }^{3}$ Division of Cancer Studies, King's College London, London, UK \\ ${ }^{4}$ Department of Epidemiology and Biostatistics, Imperial College London, London, UK
}

(Submitted 23 October 2013 - Final revision received 31 July 2014 - Accepted 25 August 2014 - First published online 27 October 2014)

\section{Abstract}

Dietary lignans, quercetin and resveratrol have oestrogenic properties, and animal studies suggest that they synergistically decrease cancer risk. A protective effect of lignans on the development of oesophageal cancer in humans has recently been demonstrated, and the present study aimed to test whether these three phytochemicals synergistically decrease the risk of oesophageal cancer. Data from a Swedish nationwide population-based case-control study that recruited 181 cases of oesophageal adenocarcinoma (OAC), 158 cases of oesophageal squamous-cell carcinoma (OSCC), 255 cases of gastro-oesophageal junctional adenocarcinoma (JAC) and 806 controls were analysed. Exposure data were collected through face-to-face interviews and questionnaires. The intake of lignans, quercetin and resveratrol was assessed using a sixty-three-item FFQ. Reduced-rank regression was used to assess a dietary pattern, and a simplified dietary pattern score was categorised into quintiles on the basis of the distribution among the control subjects. Unconditional multivariable logistic regression provided OR with $95 \% \mathrm{CI}$, adjusted for all the potential risk factors. A dietary pattern rich in lignans, quercetin and resveratrol was mainly characterised by a high intake of tea, wine, lettuce, mixed vegetables, tomatoes, and whole-grain bread and a low intake of milk. There were dose-dependent associations between simplified dietary pattern scores and all types of oesophageal cancer (all $P$ for trend $<0.05$ ). On comparing the highest quintiles with the lowest, the adjusted OR were found to be 0.24 (95\% CI $0 \cdot 12$, 0.49 ) for OAC, $0.31(95 \%$ CI $0 \cdot 15,0.65)$ for OSCC, and $0.49(95 \%$ CI $0 \cdot 28,0.84)$ for JAC. The results of the present study indicate that a dietary pattern characterised by the intake of lignans, quercetin and resveratrol may play a protective role in the development of oesophageal cancer in the Swedish population.

\section{Key words: Lignans: Quercetin: Resveratrol: Dietary patterns: Reduced-rank regression: Cancer: Oesophagus}

Over the past few decades, the incidence of oesophageal adenocarcinoma (OAC) and gastro-oesophageal junctional adenocarcinoma (JAC) has increased rapidly in many Western countries $^{(1,2)}$, while that of oesophageal squamous-cell carcinoma (OSCC) has declined ${ }^{(3)}$. Although surveillance, diagnostic measures and therapy have been developed and improved for these tumours, the overall 5-year survival remains less than $15 \%$ in Western societies ${ }^{(3)}$. This highlights the need to identify preventive measures. Dietary factors play an important role in the aetiology of oesophageal cancer, and epidemiological studies have demonstrated the protective effects of certain food groups, particularly fruits and vegetables ${ }^{(4)}$ and possibly also cereals ${ }^{(5)}$, coffee ${ }^{(6)}$ and tea ${ }^{(6)}$. All these food groups are rich sources of not only several vitamins, but also dietary phytochemicals, which have anti-carcinogenic properties ${ }^{(7)}$. Lignans, quercetin and resveratrol are the three most widely occurring phytochemicals with oestrogenic properties within the Western diet ${ }^{(8-11)}$. Due to having chemical structures similar to those of female hormones, these phytochemicals have been found to have the ability to bind to oestrogen receptors and thus exert oestrogenic effects ${ }^{(12-14)}$. Furthermore, the presence of oestrogen receptors in oesophageal tissue has been observed in in vitro studies ${ }^{(15-17)}$. This is interesting as oestrogen has been suggested to be an important factor for the low incidence of OAC and JAC in women in comparison with men, with a male:female sex ratio of up to $9: 1^{(1)}$. High intake of lignans has been found to possibly decrease the risk of OAC and JAC in humans ${ }^{(18)}$; quercetin might increase lipolysis in

Abbreviations: JAC, junctional adenocarcinoma; OAC, oesophageal adenocarcinoma; OSCC, oesophageal squamous-cell carcinoma; RRR, reduced-rank regression

*Corresponding author: Y. Lin, fax +46 8517 76280, email yulanlin1984@gmail.com 
adipocytes and induce apoptosis ${ }^{(19)}$; and resveratrol can reduce the synthesis of lipids in the liver and suppress proliferation and induce apoptosis in vitro ${ }^{(20)}$. Interestingly, a combination of lignans, quercetin and resveratrol has been found to synergistically suppress adipogenesis and induce apoptosis in an in vitro study ${ }^{(21)}$. Dietary pattern analysis captures the variation in overall dietary intake and is useful for reflecting the interaction among foods or nutrients. Recently, a hypothesis-based reduced-rank regression (RRR) analysis has been used to better identify dietary patterns ${ }^{(22)}$. In the present study, data from a Swedish nationwide case-control study using RRR were analysed with the aim of assessing whether a dietary pattern rich in lignans, quercetin and resveratrol decreases the risk of OAC, JAC and OSCC.

\section{Methods}

\section{Study subjects and study design}

Data were derived from a Swedish nationwide and populationbased case-control study, which has been described in detail previously $^{(23)}$. In brief, individuals aged $<80$ years and born and living in Sweden from 1995 to 1997 were enrolled in the study. All newly diagnosed patients with OAC or JAC and patients with OSCC born on even-numbered days were eligible to participate in the study as cases. A comprehensive organisation for rapid ascertainment of cases ensured that every potential patient throughout the country was identified shortly after the confirmation of diagnosis. This organisation included contact persons at all the 195 relevant hospital departments in Sweden, as well as the six regional tumour registries. Age (within 10 years)- and sex-matched controls were randomly selected from among the individuals in the Swedish population identified in the Swedish Register of the Total Population. Professional interviewers, who were trained to treat cases and controls in a strictly similar manner, personally interviewed all the cases and controls. The participants were asked to provide details regarding their background and relevant exposures, which have been studied in separate publications, e.g. addressing the role of gastro-oesophageal reflux ${ }^{(23)}$, obesity $^{(24)}$, tobacco smoking status ${ }^{(25)}$, alcohol consumption ${ }^{(25)}$, educational level $^{(26)}$, physical activity ${ }^{(24)}$ and Helicobacterpylori infection in the aetiology of OAC, JAC and OSCC ${ }^{(27)}$. The reflux symptoms considered in the present study referred to symptoms during the entire lifetime until 5 years before the interview. The cut-off used to define reflux was well in line with the present guidelines ${ }^{(28)}$. BMI 20 years before the interview was used to classify obesity.

\section{Ethical approval}

All six Regional Ethics Review Boards in Sweden approved the present study.

\section{Dietary intake assessment}

Dietary intake was assessed using a written validated FFQ concerning the habitual intake of sixty-three foods and beverages as recalled from 20 years before the interview. This time period was considered for dietary intake assessment to obtain a plausible induction time point between exposure and the onset of the invasive cancer ${ }^{(29)}$. The questionnaires were distributed before the interview, and the written answers were completed and other uncertainties clarified during the interview. The consumption frequency of each food item was assessed on the basis of open answers, i.e. number of times of consumption per $\mathrm{d}$, week, month or year. The intakes of lignans, quercetin and resveratrol were the exposure variables in the present study. A specific database was created with information from published analytical data for the contents of these three phytochemicals. Lignans are mainly present in whole-grain bread, berries, fruits, vegetables and flaxseed ${ }^{(8)}$. The intake of lignans was calculated by taking six precursors of mammalian lignans into account: matairesinol; secoisolariciresinol; lariciresinol; pinoresinol; syringaresinol; medioresinol ${ }^{(8,30,31-38)}$. Data on quercetin contents in onions, broccoli, lettuce, tomatoes, strawberries, apples, wine, tea and fruit juice were accumulated ${ }^{(9,39-41)}$. The major sources of resveratrol were wine, tea and berries $^{(11,41-43)}$. As green tea was effectively non-existent in the Swedish market during the exposure period of interest (mid-1970s), the intake of tea was calculated by assuming all teas consumed to be black tea. The intake of wine was estimated by assuming the wine consumed to be two-thirds red wine and one-third white wine, based on typical Swedish alcohol consumption data. Energy contents were obtained from the Swedish National Food Administration database. To calculate the intake of each food item, the consumption frequency was multiplied by sex-specific portion sizes, using data from the Swedish National Dietary Survey ${ }^{(44)}$.

\section{Statistical analyses}

To derive a dietary pattern defining the intake of lignans, quercetin and resveratrol, the statistical method RRR was employed using the partial least-squares procedure in SAS ${ }^{(22)}$. A factor score was derived using RRR - a linear combination of predictor variables (food groups or food items) that maximised the variation in response variables (lignans, quercetin and resveratrol). The number of extracted dietary pattern scores was equal to the number of response variables, i.e. three dietary pattern scores were extracted, one for each single phytochemical-related dietary pattern. The first dietary pattern score was retained for subsequent analyses because it explained most of the variation in the intake of the three phytochemicals. The total intake of all the phytochemicals was analysed as energy-adjusted densities, i.e. per $4184 \mathrm{~kJ} / \mathrm{d}$ $(1000 \mathrm{kcal} / \mathrm{d})$. To analyse less population-dependent dietary pattern variables, the original dietary pattern scores were shortened and simplified ${ }^{(45)}$. The simplified dietary pattern scores were obtained by summing unweighted standardised scores of intake for each food group, which explained most of the interindividual variation in the original dietary pattern scores. The commonly used simplified approach was used by selecting only food items with high factor loadings (i.e. $\geq|0 \cdot 2|)^{(45)}$. Factor loadings represent the correlations of the intake of

cerning the habitual intake of sixty-three foods and beverages 
each food item with the dietary pattern score. The simplified dietary pattern scores were used to evaluate the association between the dietary pattern and the risk of oesophageal cancer subtypes in logistic regression models. Dietary pattern scores were categorised into quintiles on the basis of the distribution among the control subjects. To test for linear trends across dietary pattern scores, the median scores within each quintile were used and these values were treated as a continuous variable.

Unconditional logistic regression was used to estimate OR and $95 \%$ CI. The dietary pattern score was categorised into five levels based on the quintiles of scores among the control subjects. The lowest quintile of dietary pattern score was set as the reference. Possible confounding or effect modification by aetiological factors was considered in a multivariable logistic regression model: age (categorised into <55, 55-64, 65-74, or $\geq 75$ years); sex (male or female); gastro-oesophageal reflux symptoms (yes or no); BMI (<18.5, 18.5-24.9, 25-29.9, or $\geq 30.0 \mathrm{~kg} / \mathrm{m}^{2}$ ); tobacco smoking status (never smoker, previous smoker, or current smoker of any tobacco 2 years before the interview); alcohol consumption (no alcohol, $>0-15,16-70$, or $>70 \mathrm{~g} /$ week of any alcoholic beverage); educational level $(\leq 9,10-12$, or $\geq 13$ years of formal education); physical activity (in quartiles); H. pylori infection (yes or no, assessed from blood samples); total energy intake (in quartiles, based on the distribution among the control subjects). A basic model included adjustment for only age and sex, while the fully adjusted multivariable model included all the variables listed above, selected depending on the specific subtype of oesophageal cancer analysed. The reason for this tailored adjustment is that the aetiological factors differ substantially between these cancer subtypes $^{(3)}$. Especially, reflux and obesity are the main risk factors for $\mathrm{OAC}$ and JAC and alcohol consumption and tobacco smoking status are the main risk exposure factors for OSCC ${ }^{(3)}$. Participants with more than $10 \%$ missing values in the FFQ were excluded from the analyses. The SAS statistical package (version 9.0, SAS Institute, Inc.) was used for all analyses.

\section{Results}

\section{Study participants}

Among all the 618 included cases, 189 had OAC (88\% participation rate), 167 had OSCC (73\%) and 262 had JAC (84\%). The number of control subjects was 820 (73\% participation rate). Participants with more than $10 \%$ missing values in the FFQ were excluded, leaving 181 cases of OAC, 158 cases of OSCC, 255 cases of JAC and 806 controls for the final analysis. The demographics and characteristics of the study participants are summarised in Table 1 . Owing to frequency matching, age and sex distributions were similar among the cases and controls. OAC and JAC cases had more reflux and higher BMI than controls, while OSCC cases consumed more tobacco and alcohol. All case groups had fewer years of education and a lower percentage of never-tobacco-smoking participants compared with the control group. The control group consumed more tea, lettuce, whole-grain bread and mixed vegetables and less milk when compared with the case groups $(P<0.05 ; t$ test $)$. No differences were found in the intake of tomatoes and wine. The intake of quercetin and lignans was higher in the control group than in each of the case groups $(P<0.05 ; t$ test, data not shown), while the intake of resveratrol was similar in the control and case groups ( $P=0.68 ; t$ test). The simplified dietary pattern scores were positive in the control group, but negative in all the three case groups (Table 1). All cases had a lower dietary pattern score than their matched controls $(P<0.0001 ; t$ test, data not shown).

\section{Food groups contributing to the lignan, quercetin and resveratrol pattern scores}

Tea, wine, lettuce, mixed vegetables, tomatoes, milk and whole-grain bread, with a factor loading $\geq|0 \cdot 2|$, were the most important food items contributing to the intake of dietary lignans, quercetin and resveratrol, based on the estimation using the RRR analysis, and retained in the simplified dietary pattern (Table 2). Pearson's correlation coefficients of the dietary pattern scores were slightly lower for the simplified dietary pattern than for the original dietary pattern. Both the original and simplified dietary pattern scores were strongly correlated with the intake of tea and wine, which explained most of the variation in the original dietary pattern scores. Only the intake of milk was negatively associated with the studied dietary pattern scores (Table 2).

\section{Dietary pattern characterised by the intake of lignans, quercetin and resveratrol and the risk of oesophageal cancer by histological type}

The identified dietary pattern characterised by the intake of lignans, quercetin and resveratrol was strongly associated with a decreased risk of OAC, JAC and OSCC (Table 3). Dosedependent associations were found between higher simplified dietary pattern scores and a decreased risk of each of the studied cancer subtypes (all $P$ for trend $<0.05$ ) (Table 3 ). Comparison of the extreme quintiles (quintile $5 v$. quintile 1) of scores in the fully adjusted model revealed decreased risk estimates of OAC (OR 0.24, $95 \%$ CI 0.12, 0.49), OSCC (OR 0.31, $95 \%$ CI $0 \cdot 15,0 \cdot 65)$, JAC (OR $0.49,95 \%$ CI $0 \cdot 28,0 \cdot 84)$, and OAC and JAC combined (OR 0.42, $95 \%$ CI 0.26, 0.67).

\section{Discussion}

This is the first study to define a dietary pattern characterised by the intake of the phytochemicals lignans, quercetin and resveratrol and to examine its potential association with the risk of oesophageal cancer. Strong negative associations were found between a dietary pattern rich in lignans, quercetin and resveratrol and the risk of all histological types of oesophageal cancer.

High dietary pattern scores for these three phytochemicals were mainly derived from a high intake of tea, wine, lettuce, 
Table 1. Characteristics of oesophageal cancer cases and control subjects in a Swedish population-based case-control study

(Number of subjects and percentages)

\begin{tabular}{|c|c|c|c|c|c|c|c|c|}
\hline \multirow[b]{2}{*}{ Characteristics } & \multicolumn{2}{|c|}{ Control subjects } & \multicolumn{2}{|c|}{$\begin{array}{l}\text { Oesophageal } \\
\text { adenocarcinoma } \\
\text { cases }\end{array}$} & \multicolumn{2}{|c|}{$\begin{array}{l}\text { Oesophageal } \\
\text { squamous-cell } \\
\text { carcinoma cases }\end{array}$} & \multicolumn{2}{|c|}{$\begin{array}{l}\text { Gastro-oesophageal } \\
\text { junctional } \\
\text { adenocarcinoma cases }\end{array}$} \\
\hline & $n$ & $\%$ & $n$ & $\%$ & $n$ & $\%$ & $n$ & $\%$ \\
\hline \multicolumn{9}{|l|}{ Sex } \\
\hline Male & 667 & $82 \cdot 8$ & 158 & $87 \cdot 3$ & 114 & $72 \cdot 1$ & 216 & $84 \cdot 7$ \\
\hline Female & 139 & $17 \cdot 2$ & 23 & $12 \cdot 7$ & 44 & $27 \cdot 9$ & 39 & $15 \cdot 3$ \\
\hline \multicolumn{9}{|l|}{ Age (years) } \\
\hline$<55$ & 127 & $15 \cdot 8$ & 21 & 11.5 & 13 & $8 \cdot 3$ & 52 & $20 \cdot 4$ \\
\hline $55-64$ & 185 & $23 \cdot 0$ & 38 & $20 \cdot 1$ & 58 & $36 \cdot 7$ & 57 & $22 \cdot 4$ \\
\hline $65-74$ & 317 & $39 \cdot 2$ & 88 & $48 \cdot 6$ & 62 & $29 \cdot 2$ & 105 & $41 \cdot 2$ \\
\hline$\geq 75$ & 177 & $22 \cdot 0$ & 34 & $18 \cdot 8$ & 25 & $15 \cdot 8$ & 41 & $16 \cdot 0$ \\
\hline \multicolumn{9}{|l|}{ Educational level (years) } \\
\hline$\leq 9$ & 489 & $60 \cdot 7$ & 136 & $75 \cdot 1$ & 114 & $72 \cdot 2$ & 166 & $65 \cdot 1$ \\
\hline $10-12$ & 158 & $19 \cdot 6$ & 22 & $12 \cdot 2$ & 23 & $14 \cdot 6$ & 54 & $21 \cdot 2$ \\
\hline$\geq 13$ & 159 & $19 \cdot 7$ & 23 & $12 \cdot 7$ & 21 & $13 \cdot 2$ & 35 & $13 \cdot 7$ \\
\hline \multicolumn{9}{|l|}{ BMI $\left(\mathrm{kg} / \mathrm{m}^{2}\right)$} \\
\hline$<18.5$ & 9 & $1 \cdot 1$ & 1 & 0.6 & 5 & $3 \cdot 1$ & 3 & 1.2 \\
\hline $18.5-24.9$ & 553 & $69 \cdot 0$ & 75 & 41.4 & 102 & $64 \cdot 6$ & 139 & 54.5 \\
\hline $25 \cdot 0-29 \cdot 9$ & 215 & $26 \cdot 8$ & 84 & $46 \cdot 4$ & 41 & $26 \cdot 0$ & 89 & 34.9 \\
\hline$\geq 30.0$ & 25 & 3.1 & 21 & 11.6 & 10 & $6 \cdot 3$ & 24 & 9.4 \\
\hline \multicolumn{9}{|l|}{ Tobacco smoking status } \\
\hline Never & 321 & $39 \cdot 8$ & 55 & $30 \cdot 4$ & 20 & $12 \cdot 6$ & 42 & $16 \cdot 5$ \\
\hline Ever & 309 & $38 \cdot 4$ & 84 & $46 \cdot 4$ & 42 & $26 \cdot 6$ & 121 & 47.5 \\
\hline Current & 176 & $21 \cdot 8$ & 42 & $23 \cdot 2$ & 96 & $60 \cdot 8$ & 92 & $36 \cdot 0$ \\
\hline \multicolumn{9}{|l|}{ Alcohol consumption (g/week) } \\
\hline 0 & 128 & $15 \cdot 9$ & 35 & $19 \cdot 3$ & 15 & 9.5 & 31 & $12 \cdot 2$ \\
\hline $0-15$ & 214 & $26 \cdot 5$ & 53 & $29 \cdot 2$ & 32 & $20 \cdot 3$ & 72 & $28 \cdot 2$ \\
\hline $16-70$ & 286 & 35.5 & 51 & $28 \cdot 2$ & 38 & $24 \cdot 0$ & 77 & $30 \cdot 2$ \\
\hline$>70$ & 178 & $22 \cdot 1$ & 42 & $23 \cdot 2$ & 73 & $46 \cdot 2$ & 75 & 29.4 \\
\hline \multicolumn{9}{|l|}{ Reflux } \\
\hline Yes & 131 & $16 \cdot 3$ & 110 & $60 \cdot 8$ & 25 & $15 \cdot 8$ & 74 & $29 \cdot 0$ \\
\hline No & 675 & 83.7 & 71 & $39 \cdot 2$ & 133 & $84 \cdot 2$ & 181 & $71 \cdot 0$ \\
\hline \multicolumn{9}{|l|}{ Mean daily dietary intake } \\
\hline Tea $(g)$ & \multicolumn{2}{|c|}{158.9} & \multicolumn{2}{|c|}{$95 \cdot 8$} & \multicolumn{2}{|c|}{$92 \cdot 3$} & \multicolumn{2}{|c|}{121.5} \\
\hline Wine $(g)$ & \multicolumn{2}{|c|}{$29 \cdot 9$} & \multicolumn{2}{|c|}{$20 \cdot 4$} & \multicolumn{2}{|c|}{36.5} & \multicolumn{2}{|c|}{$26 \cdot 4$} \\
\hline Lettuce $(\mathrm{g})$ & \multicolumn{2}{|c|}{$22 \cdot 6$} & \multicolumn{2}{|c|}{$15 \cdot 5$} & & & & \\
\hline Mixed vegetables $(\mathrm{g})$ & & & & & & & & \\
\hline Tomatoes $(\mathrm{g})$ & & & & & & & & \\
\hline Milk (g) & & & & & & & & \\
\hline Whole-grain bread (g) & & & & & & & & \\
\hline Lignans $(\mathrm{mg})^{\star}$ & & & & & & & & \\
\hline Quercetin (mg) & & & & & & & & \\
\hline Resveratrol (mg) & & & & & & & & \\
\hline Mean simplified dietary pattern score $†$ & & & & & & & & \\
\hline
\end{tabular}

* Including secoisolariciresinol, matairesinol, lariciresinol, isolariciresinol, pinoresinol, syringaresinol and medioresinol.

†A simplified dietary pattern score was obtained by summing standardised intake values of tea, wine, lettuce, mixed vegetables, tomatoes, milk and whole-grain bread as energy-adjusted densities.

mixed vegetables, tomatoes, and whole-grain bread and a low intake of milk.

Tea, a rich food source of quercetin and lignans in Western populations, had the highest loading factor in the dietary pattern characterised by the intake of phytochemicals, and black tea has been indicated as a factor that can prevent the development of oesophageal cancer ${ }^{(6)}$. Moreover, animal research has identified black tea to have anti-carcinogenic properties, which could be synergistically enhanced by the intake of resveratrol $^{(46)}$. Tea consumption has been shown to be a significant risk factor for OSCC in Iran, which may be mainly due to the habit of drinking tea at very high temperatures, which could cause injury to the oesophageal mucosa and increase the risk of OSCC ${ }^{(47)}$. In Sweden, where tea is not consumed at high temperatures, no associations between tea consumption and the risk of any of the main histological types of oesophageal cancer have been found ${ }^{(48)}$. Wine, which is rich in resveratrol, was another key component in the defined dietary pattern in the present study. It is possible that resveratrol might have contributed to the decreased risk of OAC and OSCC among wine drinkers observed in some studies ${ }^{(49)}$. Furthermore, high intake of fruits and vegetables has been found to decrease the risk of oesophageal cancer ${ }^{(4)}$. Fruits and vegetables are the main sources of the three studied phytochemicals ${ }^{(50)}$. Particularly, tomatoes and lettuce, the main contributors of quercetin intake, were highlighted in the defined dietary pattern. This finding parallels that of a previous study, in which tomatoes were a key component 
Table 2. Food groups mainly contributing to a high phytochemical (resveratrol, quercetin and lignans) dietary pattern score

\begin{tabular}{lcccc}
\hline & \multicolumn{2}{c}{ Original dietary pattern score* } & & Simplified dietary pattern score ${ }^{*}$ \\
\cline { 2 - 3 } Dietary intake $\neq$ & Pearson's correlation coefficient & Factor loading & & Pearson's correlation coefficient \\
\hline Tea & 0.70 & 0.48 & & 0.70 \\
Wine & 0.52 & 0.36 & \\
Lettuce & 0.39 & 0.27 & 0.71 \\
Mixed vegetables & 0.34 & 0.23 & \\
Tomatoes & 0.32 & 0.22 & \\
Milk & -0.31 & -0.21 & \\
Whole-grain bread & 0.29 & 0.20 & \\
\hline
\end{tabular}

* The original dietary pattern score was estimated using reduced-rank regression analysis.

†A simplified dietary pattern score was obtained by summing standardised intake values of tea, wine, lettuce, mixed vegetables, tomatoes, milk and whole-grain bread as energy-adjusted densities.

‡ Dietary intake is expressed as energy-adjusted densities, i.e. per $4184 \mathrm{~kJ} / \mathrm{d}(1000 \mathrm{kcal} / \mathrm{d})$.

in a 'healthy diet' pattern for preventing the development of oesophageal cancer ${ }^{(51)}$. Whole-grain bread is the most important dietary source of lignans in Sweden and other European countries $^{(52)}$. High intake of whole-grain bread has been reported to be associated with a decreased risk of OAC and has been found to be a component of a 'healthy pattern' for OAC, together with a low intake of milk $^{(53)}$. A high intake of energy from milk is usually associated with a low intake of energy from fruits and vegetables, which often results in a low intake of these three phytochemicals, and a high intake of milk might increase the risk of oesophageal cancer ${ }^{(54)}$. However, we cannot confirm that the beneficial effect of these three phytochemicals on the risk of oesophageal cancer observed in the present study is different from any accumulated or synergistic effect of phytochemicals in the plant-based diet.

Epidemiological studies examining lignans, quercetin and resveratrol separately in relation to the risk of oesophageal cancer are scarce. The oestrogenic and anti-obesity properties of lignans might have contributed to the decreased risk of oesophageal cancer found in the present study ${ }^{(8)}$. Animal research has found that quercetin can induce apoptosis ${ }^{(55)}$, but no epidemiological studies have addressed the role of quercetin intake in the development of oesophageal cancer.

Table 3. Quintiles of simplified dietary pattern scores based on the intake of food items that were derived to explain variation in the intake of the phytochemicals resveratrol, quercetin and lignans

(Odds ratios and $95 \%$ confidence intervals; number of subjects and percentages)

\begin{tabular}{|c|c|c|c|c|c|c|c|c|c|c|}
\hline & \multicolumn{9}{|c|}{ Quintiles of simplified dietary pattern scores } & \multirow[b]{3}{*}{$P$ for trend ${ }^{*}$} \\
\hline & \multirow{2}{*}{$\begin{array}{c}1 \\
\text { OR }\end{array}$} & \multicolumn{2}{|r|}{2} & \multicolumn{2}{|r|}{3} & \multicolumn{2}{|r|}{4} & \multicolumn{2}{|r|}{5} & \\
\hline & & OR & $95 \% \mathrm{Cl}$ & OR & $95 \% \mathrm{Cl}$ & OR & $95 \% \mathrm{Cl}$ & OR & $95 \% \mathrm{Cl}$ & \\
\hline \multicolumn{11}{|l|}{ Controls } \\
\hline$n$ & 161 & & 162 & & 161 & & 160 & & 162 & \\
\hline$\%$ & $20 \cdot 0$ & & $20 \cdot 1$ & & $20 \cdot 0$ & & $19 \cdot 8$ & & $20 \cdot 1$ & \\
\hline \multicolumn{11}{|c|}{ Oesophageal adenocarcinoma cases } \\
\hline$n$ & 61 & & 31 & & 42 & & 29 & & 18 & \\
\hline$\%$ & 33.7 & & $17 \cdot 1$ & & $23 \cdot 2$ & & $16 \cdot 0$ & & $10 \cdot 0$ & \\
\hline Age and sex adjusted & Reference & 0.56 & $0.31,0.82$ & 0.71 & $0.45,1.12$ & 0.48 & $0.29,0.80$ & 0.31 & $0.17,0.55$ & 0.0002 \\
\hline Multivariable adjusted $\dagger$ & Reference & 0.46 & $0.26,0.83$ & 0.69 & $0.40,1.20$ & 0.56 & $0.30,1.03$ & 0.24 & $0.12,0.49$ & 0.0007 \\
\hline \multicolumn{11}{|c|}{ Oesophageal squamous-cell carcinoma cases } \\
\hline$n$ & 40 & & 28 & & 40 & & 30 & & 20 & \\
\hline$\%$ & $25 \cdot 3$ & & $17 \cdot 7$ & & $25 \cdot 3$ & & $19 \cdot 0$ & & $12 \cdot 7$ & \\
\hline Age and sex adjusted & Reference & 0.64 & $0.37,1.10$ & 0.87 & $0.53,1.44$ & 0.59 & $0.34,1.01$ & 0.38 & $0.21,0.70$ & 0.003 \\
\hline Multivariable adjusted $\ddagger$ & Reference & 0.56 & $0.31,1.02$ & 0.81 & $0.46,1.42$ & 0.50 & $0.27,0.93$ & 0.31 & $0.15,0.65$ & 0.003 \\
\hline \multicolumn{11}{|c|}{ Gastro-oesophageal junctional adenocarcinoma cases } \\
\hline$n$ & 73 & & 56 & & 55 & & 35 & & 36 & \\
\hline$\%$ & $28 \cdot 6$ & & $22 \cdot 0$ & & $21 \cdot 6$ & & $13 \cdot 7$ & & $14 \cdot 1$ & \\
\hline Age and sex adjusted & Reference & 0.75 & $0.50,1.13$ & 0.77 & $0.51,1.16$ & 0.47 & $0.29,0.74$ & 0.45 & $0.28,0.73$ & 0.0003 \\
\hline Multivariable adjusted $\dagger$ & Reference & 0.72 & $0.46,1 \cdot 14$ & 0.75 & $0.47,1.18$ & 0.51 & $0.30,0.85$ & 0.49 & $0.28,0.84$ & 0.008 \\
\hline \multicolumn{11}{|c|}{ Oesophageal or gastro-oesophageal junction adenocarcinoma cases } \\
\hline$n$ & 134 & & 87 & & 97 & & 64 & & 54 & \\
\hline$\%$ & $30 \cdot 7$ & & $20 \cdot 0$ & & $22 \cdot 3$ & & 14.7 & & $12 \cdot 4$ & \\
\hline Age and sex adjusted & Reference & 0.64 & $0.45,0.91$ & 0.74 & $0.52,1.04$ & 0.48 & $0.33,0.69$ & 0.40 & $0.27,0.58$ & $<0.0001$ \\
\hline Multivariable adjusted $\dagger$ & Reference & 0.67 & $0.45,0.98$ & 0.74 & $0.50,1.09$ & 0.55 & $0.36,0.85$ & 0.42 & $0.26,0.67$ & 0.0005 \\
\hline
\end{tabular}

*Two-sided $P$ values for trend were calculated by the Wald statistics, using the median values for each category of dietary pattern score as a continuous variable. † Adjusted for age, sex, energy, educational level, tobacco smoking status, alcohol consumption, BMI, physical activity, reflux and Helicobacter pylori infection.

$\ddagger$ Adjusted for age, sex, energy, educational level, tobacco smoking status, alcohol consumption and physical activity. 
However, a strong inverse association between quercetin intake and distal gastric cancer risk has been reported ${ }^{(56)}$. Resveratrol can exert anti-carcinogenic effects and modulate lipid and lipoprotein metabolism and has oestrogenic properties $^{(57)}$. A dietary pattern characterised by a high combined intake of these three phytochemicals might exert an anti-carcinogenic effect based on the synergistic effect on the suppression of adipogenesis and induction of apoptosis, as indicated in animal research ${ }^{(21)}$.

The strengths of the present study include the populationbased design, including most newly diagnosed cases of the three oesophageal cancer subtypes in Sweden over 3 years, and the rigorous tumour classification of site and histological types. The random sampling of controls from the entire Swedish population reduced the possibility of biased selection. Another strength was the ability to adjust the results for all the established risk factors, counteracting bias due to confounding. Finally, the adopted RRR approach is a novel and powerful tool for describing overall dietary habits, with a focus on the variation in the intake of the interested diseaserelated nutrients. Weaknesses include information bias of exposures, as dietary intake estimations based on FFQ are typically associated with some level of measurement errors. The requirement to recall past dietary habits could result in misclassification of the exposure factors, but significant differences in the recall of dietary habits between cases and controls are not likely to occur because of their case-control (recall bias) status. In fact, we have shown in a previous validation study that cases and controls have equal reliability regarding recalling remote dietary habits ${ }^{(29)}$. Moreover, previous dietary studies based on the same case-control study on which the present study is based have reported clear differences in associations depending on the histological type of oesophageal cancer, further indicating that recall bias is not a major concern $^{(58,59)}$. The phytochemical exposure was based on the dietary intake 20 years before the interview, resulting in an increased risk of misclassification due to difficulties in recalling previous dietary habits. Furthermore, the phytochemical concentration values in the food database used in the present study were derived from a recently published paper, which might not be a good representative of foods consumed in the 1970s because of changes in industrial technology and cooking procedures. However, the cooking procedures and dietary habits have apparently not changed since the 1970s in the Swedish population, according to the Swedish National Food Administration. Moreover, this method of dietary intake assessment of food items 20 years earlier has been validated with good results and shown to capture a combination of previous and current dietary habits ${ }^{(29)}$. Non-participation was limited, but existed, which introduces a risk of selection bias. However, an association between participation and the dietary pattern studied was not likely, and thus non-participation cannot explain the findings of the present study. Finally, although the sample size was comparatively large, there were a limited number of cases in each quintile of dietary pattern scores, leaving a risk of chance errors. However, the strongly decreased risk estimates obtained in the present study were clearly statistically significant, and the dose-response trends further indicate the robustness of the findings.

In conclusion, this comprehensive and population-based case-control study indicates that a high combined dietary intake of foods rich in lignans, quercetin and resveratrol (represented by a high intake of tea, wine, tomatoes, lettuce, mixed vegetables, and whole-grain bread and a low intake of milk) could prevent the development of OAC, OSCC and JAC.

\section{Acknowledgements}

The authors thank all the 227 doctors who acted as contact persons at the participating hospital departments throughout Sweden.

The present study was supported by the Faculty Funds for Partial Financing of New Doctoral Students from Karolinska Institutet (12059012/KID-medel 2010), the Swedish Cancer Society, and the Swedish Research Council (SIMSAM).

The authors' contributions are as follows: J. L., Y. Lu and Y. Lin were responsible for the conception and design of the study; J. L. and Y. Lu obtained financial support to conduct the study; J. L. was responsible for the provision of study materials; J. L. collected and assembled the data; J. L., Y. Lu and Y. Lin analysed and interpreted the data. All authors contributed to manuscript preparation.

None of the authors has any conflicts of interest to declare.

\section{References}

1. Lagergren J \& Lagergren P (2013) Recent developments in esophageal adenocarcinoma. CA Cancer J Clin 63, 232-248.

2. Buas MF \& Vaughan TL (2013) Epidemiology and risk factors for gastroesophageal junction tumors: understanding the rising incidence of this disease. Semin Radiat Oncol 23, 3-9.

3. Lagergren J \& Lagergren P (2010) Oesophageal cancer. BMJ 341, c6280.

4. Terry P, Lagergren J, Hansen H, et al. (2001) Fruit and vegetable consumption in the prevention of oesophageal and cardia cancers. Eur J Cancer Prev 10, 365-369.

5. Terry P, Lagergren J, Ye WM, et al. (2001) Inverse association between intake of cereal fiber and risk of gastric cardia cancer. Gastroenterology 120, 387-391.

6. Tavani A, Bertuzzi M, Talamini R, et al. (2003) Coffee and tea intake and risk of oral, pharyngeal and esophageal cancer. Oral Oncol 39, 695-700.

7. Arts IC \& Hollman PC (2005) Polyphenols and disease risk in epidemiologic studies. Am J Clin Nutr 81, 317S-325S.

8. Adlercreutz H (2007) Lignans and human health. Crit Rev Clin Lab Sci 44, 483-525.

9. Hertog MGL, Hollman PCH \& Katan MB (1992) Content of potentially anticarcinogenic flavonoids of 28 vegetables and 9 fruits commonly consumed in the Netherlands. J Agric Food Chem 40, 2379-2383.

10. Peeters PHM, Slimani N, van der Schouw YT, et al. (2007) Variations in plasma phytoestrogen concentrations in European adults. J Nutr 137, 1294-1300.

11. Lamuelaraventos RM, Romeroperez AI, Waterhouse AL, et al. (1995) Direct HPLC analysis of cis-resveratrol and transresveratrol and piceid isomers in Spanish red Vitis vinifera wines. J Agric Food Chem 43, 281-283.

12. Penttinen P, Jaehrling J, Damdimopoulos AE, et al. (2007) Diet-derived polyphenol metabolite enterolactone is a 
tissue-specific estrogen receptor activator. Endocrinology 148, 4875-4886.

13. van der Woude H, ter Veld MGR, Jacobs N, et al. (2005) The stimulation of cell proliferation by quercetin is mediated by the estrogen receptor. Mol Nutr Food Res 49, 763-771.

14. Gehm BD, McAndrews JM, Chien PY, et al. (1997) Resveratrol, a polyphenolic compound found in grapes and wine, is an agonist for the estrogen receptor. Proc Natl Acad Sci U S A 94, 14138-14143.

15. Tiffin N, Suvarna SK, Trudgill NJ, et al. (2003) Sex hormone receptor immunohistochemistry staining in Barrett's oesophagus and adenocarcinoma. Histopathology 42, 95-96.

16. Moore JT, McKee DD, Slentz-Kesler K, et al. (1998) Cloning and characterization of human estrogen receptor beta isoforms. Biochem Biophys Res Commun 247, 75-78.

17. Joubert A \& Marais S (2007) In vitro effects of 2-methoxyestradiol on cell morphology and Cdc2 kinase activity in SNO oesophageal carcinoma cells. Cell Biochem Funct 25, 357-362.

18. Lin Y, Yngve A, Lagergren J, et al. (2012) Dietary intake of lignans and risk of adenocarcinoma of the esophagus and gastroesophageal junction. Cancer Causes Control 23, 837-844.

19. Kuppusamy UR \& Das NP (1994) Potentiation of beta-adrenoceptor agonist-mediated lipolysis by quercetin and fisetin in isolated rat adipocytes. Biochem Pharmacol 47, 521-529.

20. Ferry-Dumazet H, Garnier O, Mamani-Matsuda M, et al. (2002) Resveratrol inhibits the growth and induces the apoptosis of both normal and leukemic hematopoietic cells. Carcinogenesis 23, 1327-1333.

21. Park HJ, Yang JY, Ambati S, et al. (2008) Combined effects of genistein, quercetin, and resveratrol in human and 3T3-L1 adipocytes. J Med Food 11, 773-783.

22. Hoffmann K, Schulze MB, Schienkiewitz A, et al. (2004) Application of a new statistical method to derive dietary patterns in nutritional epidemiology. Am J Epidemiol 159, 935-944.

23. Lagergren J, Bergstrom R, Lindgren A, et al. (1999) Symptomatic gastroesophageal reflux as a risk factor for esophageal adenocarcinoma. $N$ Engl J Med 340, 825-831.

24. Lagergren J, Bergstrom R \& Nyren O (1999) Association between body mass and adenocarcinoma of the esophagus and gastric cardia. Ann Intern Med 130, 883-890.

25. Lagergren J, Bergstrom R, Lindgren A, et al. (2000) The role of tobacco, snuff and alcohol use in the aetiology of cancer of the oesophagus and gastric cardia. Int $J$ Cancer $\mathbf{8 5}$, $340-346$

26. Jansson C, Johansson ALV, Nyren O, et al. (2005) Socioeconomic factors and risk of esophageal adenocarcinoma: a nationwide Swedish case-control study. Cancer Epidemiol Biomarkers Prev 14, 1754-1761.

27. Ye WM, Held M, Lagergren J, et al. (2004) Helicobacter pylori infection and gastric atrophy: risk of adenocarcinoma and squamous-cell carcinoma of the esophagus and adenocarcinoma of the gastric cardia. J Natl Cancer Inst 96, 388-396.

28. Vakil N, van Zanten SV, Kahrilas P, et al. (2006) The montreal definition and classification of gastroesophageal reflux disease: a global evidence-based consensus. Am J Gastroenterol 101, 1900-1920.

29. Wolk A, Bergstrom R, Hansson LE, et al. (1997) Reliability of retrospective information on diet 20 years ago and consistency of independent measurements of remote adolescent diet. Nutr Cancer 29, 234-241.

30. Adlercreutz H \& Mazur W (1997) Phyto-oestrogens and Western diseases. Ann Med 29, 95-120.
31. Valsta LM, Kilkkinen A, Mazur W, et al. (2003) Phytooestrogen database of foods and average intake in Finland. Br J Nutr 89, Suppl. 1, S31-S38.

32. Mazur W (1998) Phytoestrogen content in foods. Baillieres Clin Endocrinol Metab 12, 729-742.

33. Mazur WM, Duke JA, Wahala K, et al. (1998) Isoflavonoids and lignans in legumes: nutritional and health aspects in humans. J Nutr Biochem 9, 193-200.

34. Mazur WM, Wahala K, Rasku S, et al. (1998) Lignan and isoflavonoid concentrations in tea and coffee. Br J Nutr 79, 37-45.

35. Mazur W, Fotsis T, Wahala K, et al. (1996) Isotope dilution gas chromatographic mass spectrometric method for the determination of isoflavonoids, coumestrol, and lignans in food samples. Anal Biochem 233, 169-180.

36. Milder IEJ, Arts ICW, van de Putte B, et al. (2005) Lignan contents of Dutch plant foods: a database including lariciresinol, pinoresinol, secoisolariciresinol and matairesinol. Br J Nutr 93, 393-402.

37. Mazur W \& Adlercreutz H (2000) Overview of naturally occurring endocrine-active substances in the human diet in relation to human health. Nutrition 16, 654-658.

38. Thompson LU, Boucher BA, Liu Z, et al. (2006) Phytoestrogen content of foods consumed in Canada, including isoflavones, lignans, and coumestan. Nutr Cancer 54, 184-201.

39. Hertog MGL, Hollman PCH \& Vandeputte B (1993) Content of potentially anticarcinogenic flavonoids of tea infusions, wines, and fruit juices. J Agric Food Chem 41, 1242-1246.

40. Hertog MGL, Hollman PCH, Katan MB, et al. (1993) Intake of potentially anticarcinogenic flavonoids and their determinants in adults in the Netherlands. Nutr Cancer Int $J \mathbf{2 0}$, 21-29.

41. Stecher G, Huck CW, Popp M, et al. (2001) Determination of flavonoids and stilbenes in red wine and related biological products by HPLC and HPLC-ESI-MS-MS. Fresenius $J$ Anal Chem 371, 73-80.

42. Lamikanra O, Grimm CC, Rodin JB, et al. (1996) Hydroxylated stilbenes in selected American wines. J Agric Food Chem 44, 1111-1115.

43. Jandera P, Skerikova V, Rehova L, et al. (2005) RP-HPLC analysis of phenolic compounds and flavonoids in beverages and plant extracts using a CoulArray detector. J Sep Sci $\mathbf{2 8}$, 1005-1022.

44. Becker W \& Riksmaten PM (1997-1998) Metod och resultatanalys (Method and Results Analysis). Uppsala: Livesmedelsverket.

45. Schulze MB, Hoffmann K, Kroke A, et al. (2003) An approach to construct simplified measures of dietary patterns from exploratory factor analysis. BrJ Nutr 89, 409-418.

46. George J, Singh M, Srivastava AK, et al. (2011) Resveratrol and black tea polyphenol combination synergistically suppress mouse skin tumors growth by inhibition of activated MAPKs and p53. PLOS ONE 6, e23395.

47. Islami F, Pourshams A, Nasrollahzadeh D, et al. (2009) Tea drinking habits and oesophageal cancer in a high risk area in northern Iran: population based case-control study. BMJ 338, b929.

48. Terry P, Lagergren J, Wolk A, et al. (2001) Drinking hot beverages is not associated with risk of oesophageal cancers in a Western population. Br J Cancer 84, 120-121.

49. Wu AH, Wan P \& Bernstein L (2001) A multiethnic population-based study of smoking, alcohol and body size and risk of adenocarcinomas of the stomach and esophagus (United States). Cancer Causes Control 12, 721-732. 
50. Brown LM, Swanson CA, Gridley G, et al. (1995) Adenocarcinoma of the esophagus - role of obesity and diet. J Natl Cancer Inst 87, 104-109.

51. Hajizadeh B, Rashidkhani B, Rad AH, et al. (2010) Dietary patterns and risk of oesophageal squamous cell carcinoma: a case-control study. Public Health Nutr 13, 1107-1112.

52. Lin Y, Wolk A, Hakansson N, et al. (2013) Validation of FFQ-based assessment of dietary lignans compared with serum enterolactone in Swedish women. Br J Nutr 109, $1873-1880$

53. Chen H, Ward MH, Graubard BI, et al. (2002) Dietary patterns and adenocarcinoma of the esophagus and distal stomach. Am J Clin Nutr 75, 137-144.

54. Bosetti C, La Vecchia C, Talamini R, et al. (2000) Food groups and risk of squamous cell esophageal cancer in northern Italy. Int J Cancer 87, 289-294.
55. Chen SS, Corteling R, Stevanato L, et al. (2012) Polyphenols inhibit indoleamine 3,5-dioxygenase-1 enzymatic activity - a role of immunomodulation in chemoprevention. Discov Med 14, 327-333.

56. Ekstrom AM, Serafini M, Nyren O, et al. (2011) Dietary quercetin intake and risk of gastric cancer: results from a population-based study in Sweden. Ann Oncol 22, 438-443.

57. Signorelli P \& Ghidoni R (2005) Resveratrol as an anticancer nutrient: molecular basis, open questions and promises. J Nutr Biochem 16, 449-466.

58. Terry P, Lagergren J, Ye WM, et al. (2001) Inverse association between intake of cereal fiber and risk of gastric cardia cancer - reply. Gastroenterology 121, 1025.

59. Terry PD, Lagergren J, Wolk A, et al. (2003) Dietary intake of heterocyclic amines and cancers of the esophagus and gastric cardia. Cancer Epidemiol Biomarkers Prev 12, 940-944. 